\title{
Synergic Effect of Combined Therapy of Hyperbaric Oxygen and Adipose-derived Mesenchymal Stem Cells on Improving Neurological Function After Acute Traumatic Spinal Cord Injury in Rat
}

\section{Tsung-Cheng Yin}

Chang Gung Memorial Hospital Kaohsiung Branch

\section{Pei-Lin Shao}

Asia University

\section{Kuan-Hung Chen}

Chang Gung Memorial Hospital Kaohsiung Branch

Kun-Chen Lin

Chang Gung Memorial Hospital Kaohsiung Branch John Y. Chiang

Chang Gung Memorial Hospital Kaohsiung Branch

Pei-Hsun Sung

Chang Gung Memorial Hospital Kaohsiung Branch

\section{Shun-Cheng Wu}

Kaohsiung Medical University

\section{Yi-Chen Li}

Chang Gung Memorial Hospital Kaohsiung Branch

\section{Hon-Kan Yip ( $\boldsymbol{V}$ han.gung@msa.hinet.net)}

Chang Gung Memorial Hospital Kaohsiung Branch https://orcid.org/0000-0002-6305-5717

\section{Mel S Lee}

Chang Gung Memorial Hospital Kaohsiung Branch

\section{Research}

Keywords: traumatic spinal cord injury, hyperbaric oxygen, mesenchymal stem cells, inflammation, cellular stress signaling

Posted Date: April 27th, 2021

DOI: https://doi.org/10.21203/rs.3.rs-437274/v1 
License: (c) (i) This work is licensed under a Creative Commons Attribution 4.0 International License. Read Full License 


\section{Abstract}

Background: This study tested whether combined hyperbaric oxygen (HBO) and allogenic adiposederived mesenchymal stem cells (ADMSCs) would be superior to either one for improving the neurological function in rat after acute traumatic spinal cord injury (TSCl) in rat.

Methods and Results: Adult-male SD rats $(n=40)$ were equally categorized into group 1 (sham-operated control), group 2 (TSCl), group 3 (TSCI + HBO for $1.5 \mathrm{~h} /$ day for 14 consecutive days after TSCl), group 4 (TSCl + ADMSCs $/ 1.2 \times 10^{6}$ cells by intravenous injection at $3 \mathrm{~h}$ and days $1 / 2$ after TSCl) and group 5 (TSCI $+\mathrm{HBO}+$ ADMSCs), euthanized and spinal-cord tissue was harvested by day 49 after TSCl. The result showed that the protein expressions of oxidative-stress (NOX-1/NOX-2), inflammatory-signaling (TLR4/MyD88/IL-1ß/TNF-a/substance-p), cell-stress signaling (PI3K/p-AKT/p-mTOR) and the voltage gated sodium channel (Nav1.3/1.8/1.9) biomarkers were highest in group 2, lowest in group 1 and significantly lower in group 5 than in groups $3 / 4$ (all $p<0.0001$ ), but they did not differ between groups $3 / 4$. The spinal cord-damaged area, the cellular levels of inflammatory/DNA-damaged (CD68+/GFAP+/ $\mathrm{Y}-\mathrm{H} 2 \mathrm{AX}+$ cells), MAPK family biomarkers (p-P38/p-JNK/p-ERK1/2) and cellular expressions of voltage gated sodium channel (Nav.1.3, Nav.1.8 and Nav.1.9 in NF200+ cells) as well as the pain facilitated cellular expressions (p-P38+/peripherin + cells, $p-J N K+/$ peripherin + cells, $p$-ERK/NF200+ cells) exhibited an identical pattern of inflammation, whereas the neurological integrity displayed an opposite pattern of inflammation among the groups (all $p<0.0001$ ).

Conclusion: Combined HBO-ADMSCs therapy offered additional benefits for protecting the neurological architectural and functional integrity against acute TSCl.

\section{Introduction}

The productive young age group is the major contributors of the traumatic spinal cord injury (TSCl) patient [1]. The impact of injury not only causes a life-long disability and productivity hindrance, but also compromises the social-interaction physically and emotionally. The annual loss in social economy due to TSCl is highly unacceptable and the incidence of the associated negative-social event is also growing [24]. Moreover, the life expectancy of TSCI patient is established to be notably reduced as their financial hardship and demanding pressure on family support [5].

An effective way to improve function of those TSCI patient is urgent and worthy, but it is regrettably still lacking, especially in consideration of the preservation of the residual neurological function and increase in the functional recovery are the two main goals for those patients. Currently, it is a formidable challenge for physicians to achieve these missions [6-9].

Cellular destruction at the instant of the application of mechanical force is known as primary injury and the subsequent death was determined during the moment of injury. Even the remainder of cells survive under the primary injury, they still doom to death because of coming consequence. Endothelial leakage, apoptosis/death as a result of vascular endothelial cell damage [10] by reactive oxygen species (ROS) 
[11] and mitochondria dysfunction [12], and cytokine release by neutrophil in situation of inflammatory reaction will further induce local water retention, cell swelling as alternation of cell-membrane water channel dysfunction $[13,14]$, which in turn causes tissue edema, produces compression pressure to the local microcirculation, and further reduce tissue perfusion, i.e., a vicious cycle formation. This vicious cycle which enhances local ischemia and amplifies inflammation, resulting in even more ROS production and cell apoptosis, well known as secondary injury, is one of the main themes to be overcome [15]. An unacceptable high prevalence (i.e., estimated to be over 60 80\%) of neuropathic pain is one of notorious outcome in TSCl patients. Regrettably, the treatment for this neuropathic pain is rarely successful [16]. Additionally, joint deformity, muscle atrophy, and bladder and bowel dysfunction always cause the resting life of TSCl patient miserable and dark.

Plentiful data from experimental and clinical trials have shown that cell therapy effectively improved ischemia-related organ dysfunction [17-20]. The underlying mechanism for improving the organ dysfunction after cell therapy has been proposed to be that the stem cells, especially those of adiposemesenchymal stem cells (ADMSCs), have capacities of anti-inflammation, immunomodulation, suppression of oxidative stress/ROS and tissue regeneration [21-23]. Interestingly, our previous studies have further demonstrated that ADMSCs therapy significantly improved the neurological function and markedly reduced infarct volume in rat after acute ischemic stroke [24, 25]. However, whether stem cell therapy for spinal cord injury would improve the outcome is still rarely addressed [26].

Hyperbaric oxygen (HBO) therapy is a traditional therapy for patients with ischemic peripheral arterial occluded disease [27-29]. The underlying mechanism of HBO therapy involved in improving ischemic organ has been proposed to be mainly through an increase of vascular wall permeability and productions of hypoxia-inducible factor-1a (HIF-1a) and stromal cell-derived factor (SDF)-1a that enhance the angiogenesis and blood flow in the ischemic area [29] as well as the mobilization of stem cell into the ischemia region [30]. Additionally, our recent study has further shown that HBO therapy rescued the rat limb from critical limb ischemia injury mainly through anti-inflammation and reduction of the oxidative stress [30]. Based on the aforementioned issues, we proposed that HBO-assisted ADMSCs might offer a synergic effect for rescuing the spinal cord function and improving the neurological outcome against acute $\mathrm{TSCl}$ in rat.

\section{Materials And Methods}

\section{Ethics}

All animal procedures were approved by the Institute of Animal Care and Use Committee at Kaohsiung Chang Gung Memorial Hospital (Affidavit of Approval of Animal Use Protocol No. 2019062504) and performed in accordance with the Guide for the Care and Use of Laboratory Animals.

Animals were housed in an Association for Assessment and Accreditation of Laboratory Animal Care International (AAALAC; Frederick, MD, USA)-approved animal facility in our hospital with controlled 
temperature and light cycles $\left(24^{\circ} \mathrm{C}\right.$ and $12 / 12$ light cycle).

\section{Animal model of acute traumatic spinal cord injury}

Pathogen-free, adult-male Sprague-Dawley (SD) rats $(\mathrm{n}=40)$ weighing 300-325 g (Charles River Technology, BioLASCO Taiwan Co. Ltd., Taiwan) were utilized in the present study. Animals were anesthetized by inhalational $2.0 \%$ isoflurane and placed in prone position on a warming pad at $37^{\circ} \mathrm{C}$ for laminectomy. Sham-operated control (SC) animals underwent opening the skin and muscle layers only, while acute $\mathrm{TSCl}$ groups received acute $\mathrm{TSCl}$ procedure. The step-by-step procedure for creating an animal model of acute TSCl is listed below:

1. The rats were anesthetized by inhalation of $2.0 \%$ isoflurane, then placed in prone position on a warming pad at $37^{\circ} \mathrm{C}$ to secure the head and shave the back hair.

2. Skin incision was carried out at the T10 T12 levels, subperiosteal dissection of the paraspinal muscle, and then a laminectomy was performed at T10 to T12 to expose the spinal cord.

3. A modified aneurysm clip was used to create an impact-compression injury of the spinal cord. The clip was left for compressing the spinal cord for 30 seconds prior to be removed by the operator.

4. After induction of the aforementioned TSCl, the muscles were sutured using 3-0 vicryl suture (Ethicon, Somerville, NJ) and the skin/muscle layers were finally closed with Michel clips.

5. The animals recovered from anaesthesia in a portable animal intensive care unit (ThermoCare ${ }^{\circledR}$ ) for 24 hours, then were allowed to recover in a cage. Bladders were expressed 3 times daily until spontaneous voiding occurred, and any hematuria or urinary tract infection was treated with ampicillin (100 mg/kg by subcutaneous administration twice daily for 5 days).

6. Post-op wound care and the motor/sensory neurologic function assessment were performed consistently.

7. Walking feature and neurological status were recorded by days $1,3,7,14,28,35,42$ and 49 after TSCl procedure.

\section{Animal grouping}

The animals ( $\mathrm{n}=40$ ) were equally categorized into group 1 [sham-operated control (only open the skin and muscle layer of spinal cord area)], group 2 (acute TSCI), group 3 [TSCl + HBO (1.5h per day for 14 consecutive days with the first time point at $3 \mathrm{~h}$ after the acute TSCl procedure), group 4 [TSCI + ADMSCs ( $1.2 \times 10^{6}$ cells/per time) by intravenous injection at $3 \mathrm{~h}$ and days 1 and 2 after acute TSCl procedure] and group 5 (TSCl + HBO + ADMSCs), respectively. Animals in each group were euthanized by day 49 after $\mathrm{TSCl}$ procedure and the spinal cord specimen in each animal was harvested for individual study.

\section{Isolation of adipose tissue for culturing mesenchymal stem cells}

For preparation of allogenic ADMSCs, additional 12 rats were utilized in the present study. The procedure and protocol have been clearly described in our previous reports [24,25]. Briefly, rats in groups 4 and 5 
were anesthetized with inhalational $2.0 \%$ isoflurane 14 days before $\mathrm{TSCl}$ procedure for harvesting the adipose tissue surrounding the epididymis. The tissue was then be cut into $<1 \mathrm{~mm}^{3}$ pieces using a pair of sharp, sterile surgical scissors. Sterile saline $\left(37^{\circ} \mathrm{C}\right)$ was added to the homogenized adipose tissue in a ratio of 3:1 (saline: adipose tissue) by volume, followed by the addition of stock collagenase solution to a final concentration of $0.5 \mathrm{U} / \mathrm{mL}$. The cells obtained were placed to the rocker for incubation. The contents of the flask were transferred to $50 \mathrm{~mL}$ tubes after digestion, followed by centrifugation at $600 \mathrm{~g}$ for 5 minutes at room temperature. The flow-through was pipetted to a $40 \mathrm{~mm}$ filter into a new $50 \mathrm{~mL}$ conical tube. The tubes were centrifuged for a third time at $600 \mathrm{~g}$ for 5 minutes at room temperature. The cells were resuspended in saline again. Isolated ADMSCs were cultured in a $100 \mathrm{~mm}$ diameter dish with $10 \mathrm{~mL}$ DMEM culture medium containing $10 \%$ FBS for 14 days.

\section{Basso, Beattie, Bresnahan (BBB) functional scale for assessment of the locomotor capacity of rats after acute TSCI}

The animals were followed for 7 weeks (i.e., 49 days) after acute TSCI procedure. The procedure and protocol of BBB scale were based on the previous reports [31, 32]. In detail, after the aforementioned period each rat was placed in an $80 \times 80 \times 30 \mathrm{~cm}^{3}$ clear box lined with a blue non-slippery material and stimulated to move freely. Their movement was video-recorded. Identical copies of the edited videos were given to two independent evaluators who were blinded with regards to the degree of injury severity. Each evaluator made a determination of the locomotor capacity of the rats using the BBB functional scale. Finally, the parameters in each rat were calculated by averaging scores collected from these two evaluators.

\section{Hyperbaric oxygen (HBO) therapy}

The procedure and protocol of HBO therapy were based on a recent report [30]. Briefly, to induce tissuelevel hyperoxia, SD rats were subjected to HBO administration in an animal tabletop chamber (PiersolDive, model 4934) with the animals exposed to $100 \%$ oxygen at 2.4 atmospheres absolute (ATA) for 90 minutes one session for 14 consecutive days.

\section{Western blot analysis}

The spinal cord from the rats of the sham control and experimental groups were harvested as previously described [36]. Equal amounts $(50 \mu \mathrm{g})$ of protein extracts were loaded and separated by SDS-PAGE using 8-12\% acrylamide gradients. After electrophoresis, the separated proteins were transferred electrophoretically to a polyvinylidene difluoride (PVDF) membrane (Amersham Biosciences). Nonspecific sites were blocked by incubation of the membrane in blocking buffer [ $5 \%$ nonfat dry milk in T-TBS (TBS containing $0.05 \%$ Tween 20 )] overnight. The membranes were incubated with the indicated primary antibodies [NADPH oxidase (NOX)-1 (1: 2000, Sigma), NOX-2 (1:750, Sigma), toll like receptor (TLR)-4 (1:1000, Novus Biologicals), myelin and lymphocyte (MAL) (1:1000, Invitrogen Antibodies), myeloid differentiation primary response 88 (MyD88) (1:2000, Abcam), TNF receptor associated factor (TRAF) 6 (1:1000, Abcam), IKB kinase a (IKK-a) (1:1000, Abcam), IKK-ß (1:1000, Cell Signaling), interleukin (IL)-1 $\beta$ 
(1:1000, Cell Signaling), tumor necrosis factor (TNF)-a (1:1000, Cell Signaling), nuclear factor (NF)-kB (1:600, Abcam), substance $p$ (1:1000, Invitrogen Antibodies), PI3K (1:1000, Cell Signaling), phosphorylated (p)-AKT (1:1000, Cell Signaling), p-m-TOR (1:1000, Cell Signaling), Nav 1.3 (1:200, Alomone Labs), Nav 1.8 (1:2000, Abcam), Nav 1.9 (1:200, Alomone Labs) and actin (1:10000, Millipore)] for 1 hour at room temperature. Horseradish peroxidase-conjugated anti-rabbit immunoglobulin IgG (1:2000, Cell Signaling) was used as the secondary antibody for one hour incubation at room temperature. The washing procedure was repeated eight times within an hour, and immunoreactive bands were visualized by enhanced chemiluminescence (ECL; Amersham Biosciences) after exposure to Biomax L film (Kodak). For quantification, ECL signals were digitized using Labwork software (UVP).

\section{Immunofluorescent (IF) staining}

IF staining proceeded as we previously reported [36]. Rehydrated paraffin sections were first treated with $3 \% \mathrm{H} 2 \mathrm{O} 2$ for 30 minutes and incubated with Immuno-Block reagent (BioSB, Santa Barbara, CA, USA) for 30 minutes at room temperature. Sections were then incubated with primary antibodies specifically against CD68 (1:500, Abcam), glial fibrillary acidic protein (GFAP) (1:500, Dako), $\mathrm{Y}-\mathrm{H} 2 \mathrm{AX}$ (1:1000, Abcam), p-JNK (1:500, R\&B systems), p-ERK (1:200, Abcam), p-p38 (1:500, Gene Tex), NF-200 (7.5mg, Abcam), Nav1.3 (1:100, GeneTex), Nav1.8, Nav1.9 (1:100, GeneTex) and peripherin (1:1000, Abcam). Sections incubated with irrelevant antibodies served as controls. Three sections of DRG specimens were analysed in each rat. For quantification, three randomly selected high-power fields (HPFs) were analysed per section. The mean number of positively stained cells per HPF for each animal was determined across all nine HPFs.

\section{Histological quantification of spinal cord injured area}

The H.E. stain was used for identifying the area of spinal cord injury. The spinal cord specimens were sectioned at $4 \mu \mathrm{m}$ for light microscopy for each animal. Three spinal cord sections from each rat were analyzed and three randomly selected high-power fields (HPFs; 10x) were examined in each section. The integrated area $\left(\mu \mathrm{m}^{2}\right)$ of spinal cord injury on each section was calculated using the Image Tool 3 (IT3) image analysis software (University of Texas, Health Science Center, San Antonio, UTHSCSA; Image Tool for Windows, Version 3.0, USA). Three randomly selected HPFs (10x) were analyzed in each section. After assessment of the number of pixels in each injured area per HPF, the numbers of pixels obtained from three HPFs were summed. The procedure was repeated in two other sections for each animal. The mean pixel number per HPF for each animal was then calculated by summing up all pixel numbers and divided by 9 . The mean integrated area $\left(\mu \mathrm{m}^{2}\right)$ (i.e., the morphological destructive area) of per HPF was obtained using a conversion factor of 19.24 ( $1 \mu \mathrm{m}^{2}$ corresponded to 19.24 pixels).

\section{Statistical Analysis}

Quantitative data were expressed as mean \pm SD. Statistical analysis was adequately performed by one way ANOVA followed by Bonferroni multiple-comparison post hoc test. Statistical analysis was 
performed using SAS statistical software for Windows version 8.2 (SAS institute, Cary, NC, USA). A probability value $<0.05$ was considered statistically significant.

\section{Results}

\section{The time courses of neurological function after TSCl procedure (Figure 1)}

The BBB functional scale for assessment of the locomotor capacity of rats was performed for each rat on days 1, 3, 7, 14, 28, 35, 42 and 49 after acute TSCI induction. By days 1 and 3, the BBB score of left or right lower limb was significantly higher in group 1 (i.e., SC) than in groups 2 (i.e., TSCI), 3 (i.e., TSCl + $\mathrm{HBO}$ ), 4 (i.e., $\mathrm{TSCl}+\mathrm{ADMSCs}$ ) and 5 (i.e., $\mathrm{TSCl}+\mathrm{HBO}+\mathrm{ADMSCs}$ ), but it did not differ among groups 2 to 5 at these time points. Additionally, by days 7 to 49 (i.e., the end of study period), this parameter was still persistently higher in group 1 than that of those groups 2 to 5 with respect to either left or right lower limb. However, as compared with groups 2 and 4, this parameter of left lower limb was significantly higher in groups 3 and 5 and significantly higher in group 5 than in group 3 but it showed no difference between groups 2 and 4 . On the other hand, this parameter of right lower limb was significantly higher in groups 3 and 5 than in groups 2 and 4, but it was similar between the former two and between the later two groups. By days 14 to 28 , this parameter of left and right lower limbs was further significantly improved in groups 3 to 5 than in that of group 2 .

Furthermore, this BBB score was significantly higher in group 5 than in that of groups 3 and 4 after day 28 , but it did not differ between groups 3 and 4 up to the time point of day 49 after acute TSCI procedure. These findings implicated the attainment of a steady state of neurological functional improvement after days 7 to 49 following TSCl procedure among groups 3, 4 and 5 animals as compared with group 2 . Additionally, the combined therapy was found to be superior to either one alone for improvement of neurological function after TSCI procedure.

The protein expressions of oxidative stress and upstream/downstream inflammatory signalings in spinal cord tissue by day 49 after TSCl procedure (Figure 2)

To further elucidate the oxidative stress and inflammatory signaling pathways, the Western blot was utilized in the present study. The result demonstrated that the protein expressions of NOX-1 and NOX-2, two inflammatory indicators, were highest in group 2, lowest in group 1, significantly lower in group 5 than in groups 3 and 4 and lower in group 3 than in group 4. Additionally, the protein expressions of TLR4, Mal, MyD88, TRAF6, IKK-a, IKK-ß, NF-KB, IL-1ß, TNF-a and substance-p, 10 indices of up- and downstream inflammatory signaling pathways, displayed an identical pattern of oxidative stress among the groups.

The protein expressions of cell-stress signaling and voltage gated sodium channel in spinal cord tissue by day 49 after $\mathrm{TSCl}$ procedure (Figure 3 ) 
We also utilized the Western blot to evaluate the cell stress signaling in the current study. As we expected, the protein expressions of PI3K, phosphorylated (p)-AKT and p-mTOR, three indicators of cell stress signaling, and the protein expressions of Nav1.3, Nav1.8 and Nav1.9, three indicators of the voltage gated sodium channel, were highest in group 2, lowest in group 1, significantly lower in group 5 than in groups 3 and 4 and lower in group 3 than in group 4.

\section{The cellular levels of inflammationin spinal cord tissue by day 49 after TSCl procedure (Figure 4)}

Not only the protein level but also the cellular level of inflammation were determined in the present study. The IF microscopic finding demonstrated that the cellular expressions of CD68 and GFAP, two indicators of inflammation, and cellular expression of $\mathrm{Y}-\mathrm{H} 2 \mathrm{AX}$, an indicator of DNA-damaged biomarker, were highest in group 2, lowest in group 1, significantly lower in group 5 than in groups 3 and 4 and lower in group 3 than in that of group 4 .

The cellular expressions of MAPK family biomarkersin spinal cord tissue by day 49 after TSCI procedure (Figures 5, 6 and 7)

The expressions of p-P38 (Fig. 5) and p-JNK (Fig. 6) in peripherin+ cells (i.e., double staining), and ERK1/2 (Fig. 7) in NF200+ cells, three indicators of pain facilitated cellular expressions, were highest in group 2, lowest in group 1, significantly lower in group 5 than in groups 3 and 4 and lower in group 3 than in group 3.

Thecellular expressions of voltage gated sodium channel in spinal cord tissue by day 49 after TSCI procedure (Figures 8,9 and 10)

It is well recognized that voltage gated sodium channel plays an essential role for ectopic discharges or activities in response to stimulation/damage [33, 34]. In the present study, we found that the expressions of Nav1.3 (Fig. 8), Nav1.8 (Fig. 9) and Nav1.9 (Fig. 10) colocalized with NF200+ cells, three indicators of voltage gated sodium channel, were highest in group 2, lowest in group 1, significantly lower in group 5 than in groups 3 and 4 and lower in group 3 than in group 4.

\section{The spinal cord damaged areaby day 49 after TSCI procedure (Figure 11)}

Finally, the microscopic finding of H.E. stain revealed that the spinal cord damaged area (longitudinal sections) was highest in group 2, lowest in group 1, significantly lower in group 5 than in groups 3 and 4 and significantly lower group 3 than in group 4.

\section{Discussion}

This study which investigated the therapeutic impact of HBO-ADMSCs on protecting the neurologic function (i.e., locomotor capacity) and architectural integrity of spinal cord yielded several striking implications. Frist, we successfully created an animal model of acute TSCl that was reproducible for different treatment strategies. Second, the underlying mechanism of how TSCl acting on damaging the 
spinal cord had been clearly delineated. Third, we proved that combined HBO-ADMSCs was superior to either HBO or ADMSCs for protecting the integrities of spinal cord architecture and neurological function, suggesting the combined therapy offered a synergic effect.

Currently, an effective way to improve neurological function in patients after $\mathrm{TSCl}$ is still regrettably lacking, suggesting that it remains a formidable challenge for physicians to achieve this mission [6-9]. The most important finding in the present study was that the locomotor capacity (i.e., evalauted by BBB score) was significantly impaired in TSCI group than in SC counterpart. However, as comapared with $\mathrm{TSCl}$ only, this parameter was remarkably recovered in TSCl animals after receiving HBO or ADMSCs and furthermore remarkably recovered in HBO-ADMSCs treatment, suggesting that combined HBO-ADMSCs offered additional benefits for the animals. When looking at the integrity of architecture of spinal cord undergoing the microsocpic examination, we found that the anatomical structure of the spinal cord remarkably devastated in TSCl group than in SC group, while notably preserved in HBO or ADMSCs treated and moreover in HBO-ADMSCs treated TSCI animals. This finding could, at least in part, explain why the neurological function was significantly improved in that of HBO-ADMSCs treated animals and highlight that this combined therapy may pose as a therapeutic potential for the TSCl patients, especially those who are refractory to conventional therapy.

An association between organ damage and vigorous inflamamtory reaction as well as generation of oxidaitve stress which in turn, causes furthermore organ/tissue damage, resulting in organ failure has been well investigated [20-25]. In the present study, we found that the cellular-molecular levels of inflamamtory biomarkers were substantially increased in TSCl animals than in those of the $\mathrm{SC}$ animals. In this way, our findings were consistent with the findings of the previous studies [20-25]. Of distnictive finding was that HBO or ADMSCs therapy markedly supressed and combined these two regimens further markedly supressed these molecular-cellular pertubations. Of particularly distinctive finding was that not only the successful treatment was identified but also the upstream and downstream inflammatory signalings that participated in the spinal cord damage were clearly clarified in the present study (refer to Fig. 12). Accordingly, our finding highlights that the identified signaling pathway may be served as an useful reference when some medications will be considered as an accessory strategic management for those TSCI patients who are refractory to traditional management.

Cell stress signlaing is commonly elicited in setting of ischemia-reperfusion (IR) condition that has been identified to be strongly predictive of unfavorable outcome after organ IR injury [35]. An essential finding in the present study was that as compared with the SC group, the cell stress signaling was remarkably upregulated in TSCl group. In this way, our finding was consistent with the finding of previous study [35]. Intriguingly, the expression of this signaling pathway was significantly downregulated by HBO or ADMSCs therapy and further significantly downregulated by combining these two regimens.

It is well recognized the protein and cellular levels of voltage gated sodium channel [36] and those of members of mitogen activated protein kinases (MAPKs), such as p38, ERK, and JNK (i.e., MAPK family) [37], have all participated in the pain propagation and facilitation of pain sensation. A principal finding in 
the present study was that these aforementioned parameters were notably increased in TSCl animals than in those of SC animals. The results of our and previous [36,37] studies could explain why the majority of TSCl patients frequently encounter intractable pain in their lives. Of important finding in the present study was that those parameters of voltage gated sodium channel and MAPK family in injured spinal cord were significantly attenuated by HBO or ADMSCs therapy and further significantly attenuated by combined HBO-ADMSCs treatment. Our findings could be considered as an alternative strategic management for those TSCl patients suffering intractable pain refractory to conventional management.

\section{Study limitation}

Despite the results were attractive and promising, our study has limitations. First, without stepwise titrating the dose of ADMSCs and the duration of HBO therapy, we did not know which dosage/duration were the most suitable for the animals. Second, the data showed that HBO therapy appeared to be better than that of the ADMSCs therapy. However, perhaps the different duration of therapy might be one of the reasons to explain this phenomenon. Third, despite the neurological function was notably improved by day 49 , i.e., the end of the study period, the long-term outcome of these strategic management remains uncertain.

In conclusion, the results of the present study demonstrated that HBO-ADMSCs therapy effectively protected the spinal cord against acute TSCl in rodent.

\section{Abbreviations}

HBO: hyperbaric oxygen; ADMSCs: adipose-derived mesenchymal stem cells; TSCl: acute traumatic spinal cord injury; ROS: reactive oxygen species; HIF-1a: hypoxia-inducible factor-1a; SDF-1a: stromal cellderived factor; SD: Sprague-Dawley 0; SC: Sham-operated control; BBB: Basso, Beattie, Bresnahan; PVDF: polyvinylidene difluoride; TBS containing $0.05 \%$ Tween 20: T-TBS; ECL: enhanced chemiluminescence; IF: Immunofluorescent; HPFs: high-power fields; IT3: Image Tool 3; IR: ischemia-reperfusion; MAPKs: mitogen activated protein kinases

\section{Declarations}

\section{Acknowledgments}

This study was supported by a program grant from Chang Gung Memorial Hospital, Chang Gung University (Grant number: CMRPG8J0641).

\section{Author Contributions}

Investigation, Tsung-Cheng Yin, Pei-Lin Shao, Kuan-Hung Chen, Kun-Chen Lin, John Y. Chiang, Pei-Hsun Sung, Shun-Cheng Wu, Yi-Chen Li, Yi-Chen Li, Hon-Kan Yip, and Mel S. Lee; Methodology, Tsung-Cheng Yin, Pei-Lin Shao, Kuan-Hung Chen, Kun-Chen Lin, John Y. Chiang, Pei-Hsun Sung, Shun-Cheng Wu, Yi- 
Chen Li, Yi-Chen Li, Hon-Kan Yip, and Mel S. Lee; Supervision, Tsung-Cheng Yin, Hon-Kan Yip, and Mel S. Lee; Writing - review \& editing, Tsung-Cheng Yin, Hon-Kan Yip, and Mel S. Lee.

\section{Funding}

This study was supported by a program grant from Chang Gung Memorial Hospital, Chang Gung University (Grant number: CMRPG8J0641).

\section{Availability of data and materials}

The data that support the findings of this study are available from the corresponding authors upon reasonable request.

\section{Ethics approval and consent to participate}

All animal experimental procedures were approved by the Institutional Animal Care and Use Committee at Kaohsiung Chang Gung Memorial Hospital (Affidavit of Approval of Animal Use Protocol No. 2019062504) and performed in accordance with the Guide for the Care and Use of Laboratory Animals, $8^{\text {th }}$ edition (NIH publication No. 85-23, National Academy Press, Washington, DC, USA, revised 2011).

\section{Consent for publication}

Not applicable.

\section{Conflicts of interest}

All authors have read the journal's policy on disclosure of potential conflicts of interest and the journal's authorship agreement. The authors declare that they have no conflicts of interest. The article has been reviewed by and approved by all named authors.

\section{References}

1. Singh, A., et al., Global prevalence and incidence of traumatic spinal cord injury. Clin Epidemiol, 2014. 6: p. 309-31.

2. Webb, S.B., Jr., M.E. Lorenzi, and E. Berzins, Marital, educational, employment, income and general financial status prior to and one to six years post-spinal cord injury. Paraplegia, 1982. 20(2): p. 108-9.

3. Levi, R., C. Hultling, and A. Seiger, The Stockholm spinal cord injury study: 4. Psychosocial and financial issues of the Swedish annual level-of-living survey in SCI subjects and controls. Paraplegia, 1996. 34(3): p. 152-7.

4. Dismuke, C.E., et al., Diabetes increases financial burden of individuals with traumatic spinal cord injury (TSCI). Spinal Cord, 2015. 53(2): p. 135-8.

5. Fekete, C., et al., Is financial hardship associated with reduced health in disability? The case of spinal cord injury in Switzerland. PLoS One, 2014. 9(2): p. e90130. 
6. Oyinbo, C.A., Secondary injury mechanisms in traumatic spinal cord injury: a nugget of this multiply cascade. Acta Neurobiol Exp (Wars), 2011. 71(2): p. 281-99.

7. Young, W., Secondary injury mechanisms in acute spinal cord injury. J Emerg Med, 1993. 11 Suppl 1: p. 13-22.

8. Raspa, A., et al., Recent therapeutic approaches for spinal cord injury. Biotechnol Bioeng, 2016. 113(2): p. 253-9.

9. Sabapathy, V., G. Tharion, and S. Kumar, Cell Therapy Augments Functional Recovery Subsequent to Spinal Cord Injury under Experimental Conditions. Stem Cells Int, 2015. 2015: p. 132172.

10. Matsushita, T., et al., Diffuse and persistent blood-spinal cord barrier disruption after contusive spinal cord injury rapidly recovers following intravenous infusion of bone marrow mesenchymal stem cells. Exp Neurol, 2015. 267: p. 152-64.

11. Kubota, K., et al., Myeloperoxidase exacerbates secondary injury by generating highly reactive oxygen species and mediating neutrophil recruitment in experimental spinal cord injury. Spine (Phila Pa 1976), 2012. 37(16): p. 1363-9.

12. Wingrave, J.M., et al., Early induction of secondary injury factors causing activation of calpain and mitochondria-mediated neuronal apoptosis following spinal cord injury in rats. J Neurosci Res, 2003. 73(1): p. 95-104.

13. Hu, A.M., et al., Myelotomy reduces spinal cord edema and inhibits aquaporin-4 and aquaporin-9 expression in rats with spinal cord injury. Spinal Cord, 2015. 53(2): p. 98-102.

14. Zu, J., et al., Curcumin improves the recovery of motor function and reduces spinal cord edema in a rat acute spinal cord injury model by inhibiting the JAK/STAT signaling pathway. Acta Histochem, 2014. 116(8): p. 1331-6.

15. Beattie, M.S., Inflammation and apoptosis: linked therapeutic targets in spinal cord injury. Trends Mol Med, 2004. 10(12): p. 580-3.

16. D'Angelo, R., et al., Neuropathic pain following spinal cord injury: what we know about mechanisms, assessment and management. Eur Rev Med Pharmacol Sci, 2013. 17(23): p. 3257-61.

17. Nanjundappa A, Raza JA, Dieter RS, et al. Cell transplantation for treatment of left-ventricular dysfunction due to ischemic heart failure: from bench to bedside. Expert Rev Cardiovasc Ther 2007; 5: 125-131.

18. Leu S, Sun CK, Sheu JJ, et al. Autologous bone marrow cell implantation attenuates left ventricular remodeling and improves heart function in porcine myocardial infarction: an echocardiographic, sixmonth angiographic, and molecular-cellular study. Int J Cardiol 2011; 150: 156-168.

19. Zimmet H, Porapakkham P, Porapakkham P, et al. Short- and long-term outcomes of intracoronary and endogenously mobilized bone marrow stem cells in the treatment of ST-segment elevation myocardial infarction: a meta-analysis of randomized control trials. Eur J Heart Fail 2012; 14: $91-$ 105.

20. Lee FY, Chen YL, Sung PH, et al. Intracoronary Transfusion of Circulation-Derived CD34+ Cells Improves Left Ventricular Function in Patients With End-Stage Diffuse Coronary Artery Disease 
Unsuitable for Coronary Intervention. Crit Care Med 2015; 43: 2117-2132.

21. Yip HK, Lee MS, Sun CK, et al. Therapeutic effects of adipose-derived mesenchymal stem cells against brain death-induced remote organ damage and post-heart transplant acute rejection. 2017; 8:108692-108711

22. Chang CL, Sung PH, Chen KH, et al. Adipose-derived mesenchymal stem cell-derived exosomes alleviate overwhelming systemic inflammatory reaction and organ damage and improve outcome in rat sepsis syndrome. Am J Transl Res. 2018; 10:1053-1070

23. Sheu JJ, Lee MS, Wallace CG, et al. Therapeutic effects of adipose derived fresh stromal vascular fraction-containing stem cells versus cultured adipose derived mesenchymal stem cells on rescuing heart function in rat after acute myocardial infarction. Am J Transl Res. 2019; 11:67-86

24. Leu S, Lin YC, Yuen CM, et al. Adipose-derived mesenchymal stem cells markedly attenuate brain infarct size and improve neurological function in rats. J Transl Med. 2010; 8:63.

25. Chen $\mathrm{KH}$, Chen $\mathrm{CH}$, Wallace $\mathrm{CG}$, et al. Intravenous administration of xenogenic adipose-derived mesenchymal stem cells (ADMSC) and ADMSC-derived exosomes markedly reduced brain infarct volume and preserved neurological function in rat after acute ischemic stroke. 2016; 7:74537-74556

26. Cheng, I., et al., Local versus distal transplantation of human neural stem cells following chronic spinal cord injury. Spine J, 2015.

27. Sheffield PJ. How the Davis 2.36 ATA wound healing enhancement treatment table was established. Undersea Hyperb. Med. 2004; 31:193-194

28. Slovut DP, Sullivan TM. Critical limb ischemia: medical and surgical management. Vasc. Med. 2008; 13:281-291

29. Thom SR. Hyperbaric oxygen: its mechanisms and efficacy. Plast. Reconstr. Surg. 2011; 127 Suppl $1: 131 S-141 S$

30. Shan-Ling Hsu SL, Yin TH, Shao PL, et al. Hyperbaric oxygen facilitates the effect of endothelial progenitor cell therapy on improving outcome of rat critical limb ischemia. AJTR 2019 (in press)

31. Basso DM, Beattie MS, Bresnahan JC. A sensitive and reliable locomotor rating scale for open field testing in rats. J Neurotrauma. 1995;12:1-21

32. Tarcisio Eloy Pessoa de Barros Filho, et al. Analysis of the sensitivity and reproducibility of the Basso, Beattie, Bresnahan (BBB) scale in Wistar rats. Clinics (Sao Paulo) 2008 Feb;63(1):103-8.

33. Wood JN, Boorman JP, Okuse K and Baker MD. Voltage-gated sodium channels and pain pathways. J Neurobiol 2004; 61: 55-71.

34. Lai J, Porreca F, Hunter JC and Gold MS. Voltage-gated sodium channels and hyperalgesia. Annu Rev Pharmacol Toxicol 2004; 44: 371-397.

35. ChaiHT, Sheu JJ, Chiang JY, et al. Early administration of cold water and adipose derived mesenchymal stem cell derived exosome effectively protects the heart from ischemia-reperfusion injury. Am J Transl Res 2019; 11(9):5375-5389. 
36. Chien-Hui Yang, Hon-Kan Yip, et al. Long-term Therapeutic Effects of Extracorporeal Shock WaveAssisted Melatonin Therapy on Mononeuropathic Pain in Neurochem Res 2019 Apr;44(4):796-810.

37. Ji RR, Woolf CJ (2001) Neuronal plasticity and signal transduction in nociceptive neurons: implications for the initiation and maintenance of pathological pain. Neurobiol Dis 8:1-10

\section{Figures}
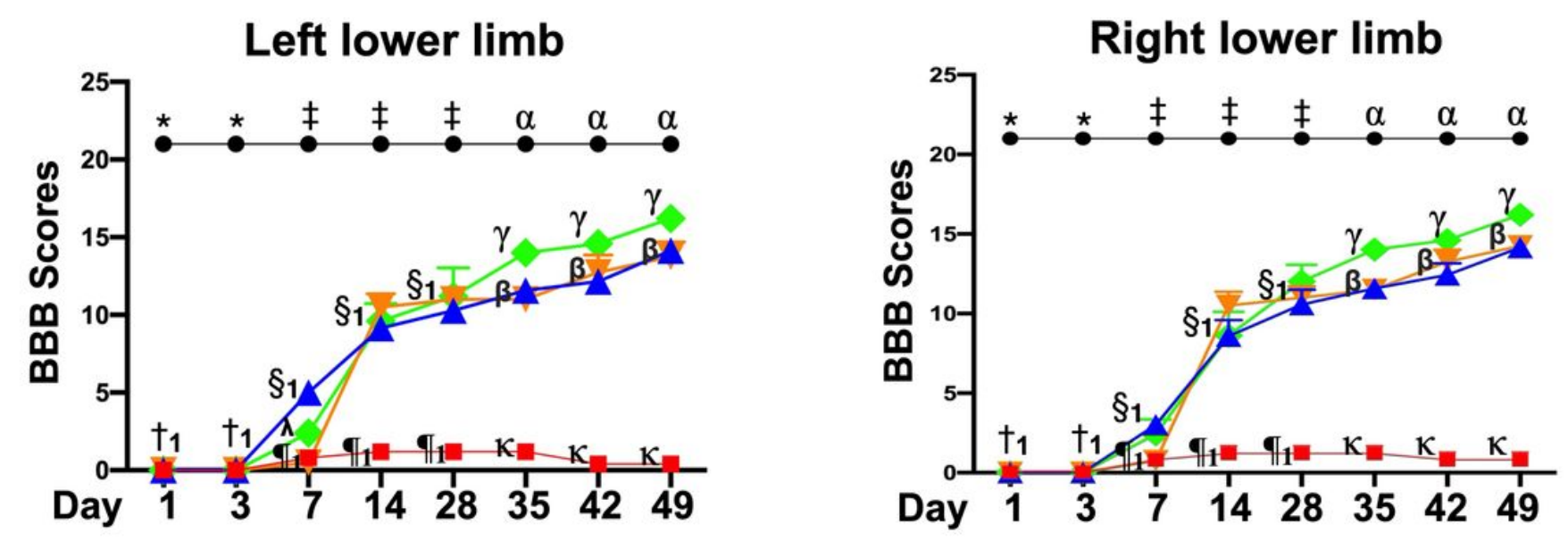

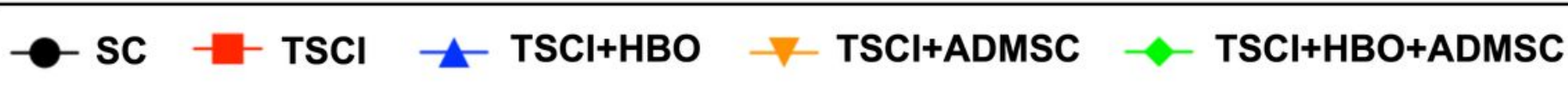

Figure 1

Time courses of BBB score among the five groups after acute TSCI procedure A) By days 1 and $3: \nabla v s . \dagger$, $p<0.0001$; symbol + indicated the $p$ value $>0.5$ among the groups of $\mathrm{SC}, \mathrm{TSCl}, \mathrm{TSCl}+\mathrm{HBO}, \mathrm{TSCl}+$ ADMSCs and TSCI + HBO + ADMSCs at these time points with respect to both right and left lower limbs.

B) By days 7, 14 and 28: $\ddagger$ vs. other groups with different symbols $(\S, 9), p<0.0001$; $\S$ indicated the $p$ value $>0.5$ among the groups TSCI + HBO, TSCl + ADMSCs and TSCI + HBO + ADMSCs at these time points with respect to both right and left lower limbs. C) By days 35, 42 and 49: a vs. other groups with different symbols $(B, \gamma, K), p<0.0001$; $\gamma$ indicated the $p$ value $>0.5$ between the groups $\mathrm{TSCl}+\mathrm{HBO}$ and $\mathrm{TSCl}+$ ADMSCs at these time points with respect to both right and left lower limbs. All statistical analyses were performed by one-way ANOVA, followed by Bonferroni multiple comparison post hoc test $(n=8$ for each group). Symbols ( $¥, \S, 9)$ or ( $a, B, \gamma, k$, ) indicate significance (at 0.05 level). SC = sham-operated control; $\mathrm{TSCl}=$ traumatic spinal cord injury; $\mathrm{HBO}$ = hyperbaric oxygen; $\mathrm{ADMSC}$ = adipose-derived mesenchymal stem cells. 
(A)
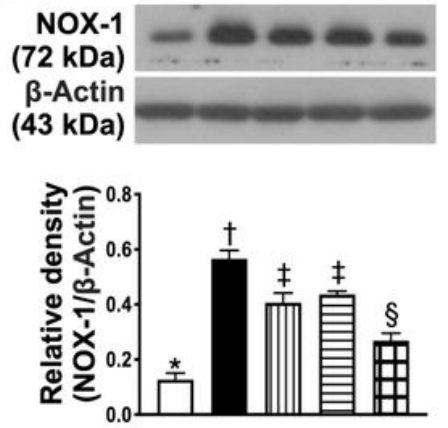

(E)
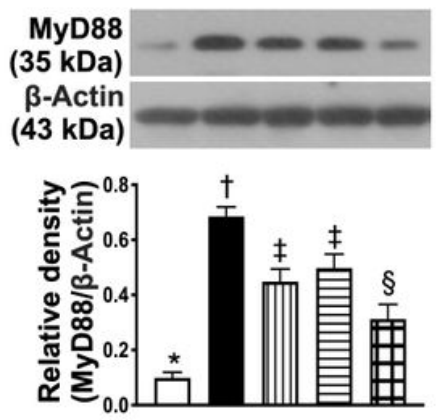

(I)
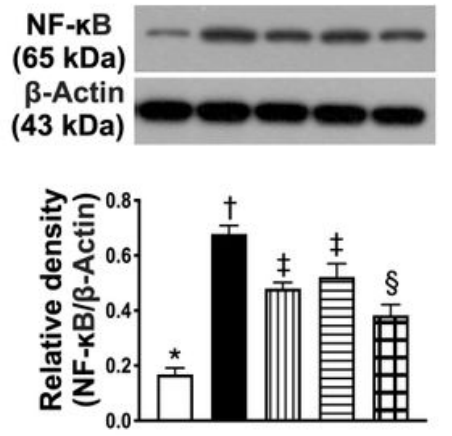

(B)
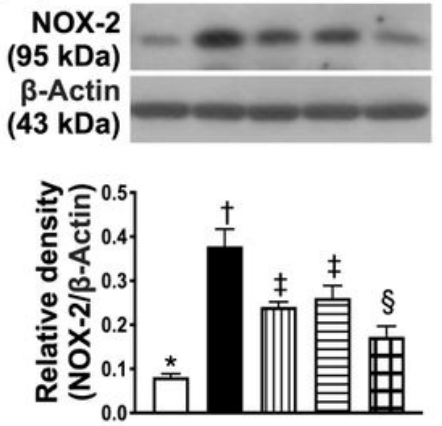

(F)
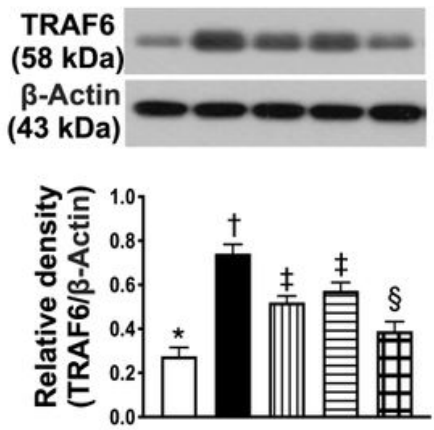

(J)

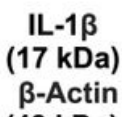

(43 kDa)

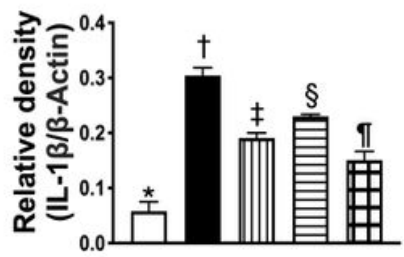

(C)
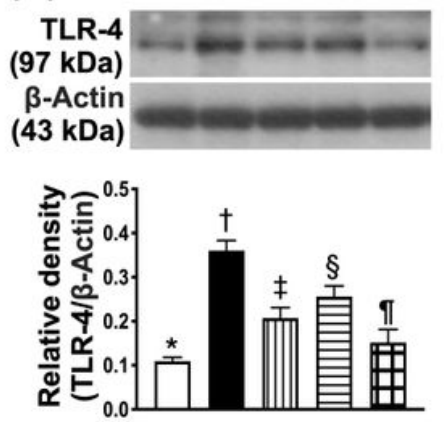

(G)
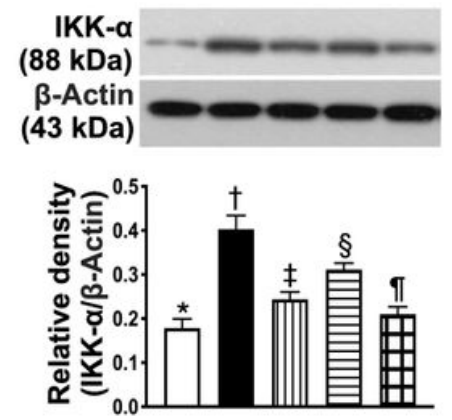

(K)
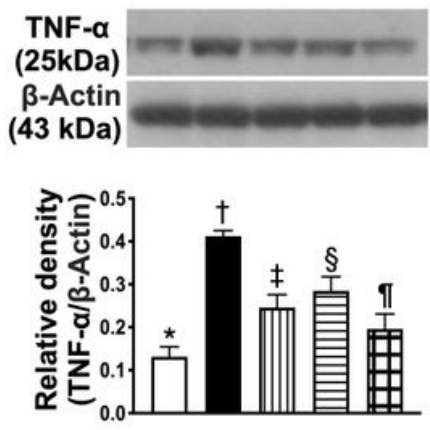

(D)

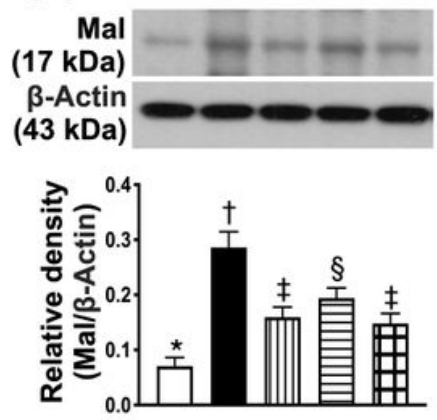

(H)
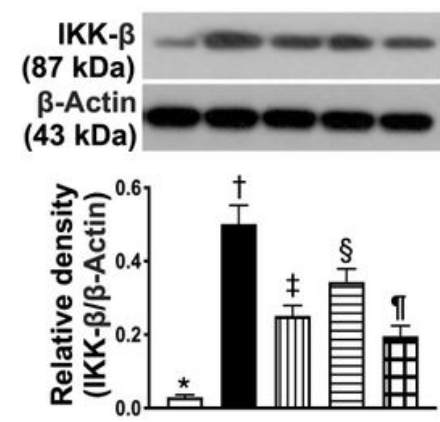

(L)

Substance-p
$(15 \mathrm{kDa})$
$\beta-A c t i n$
$(43 \mathrm{kDa})$

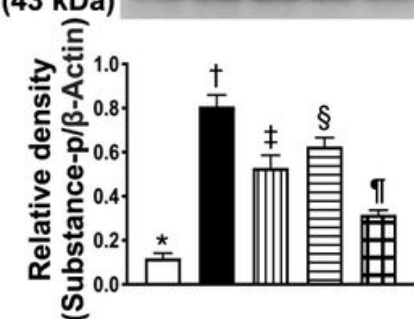

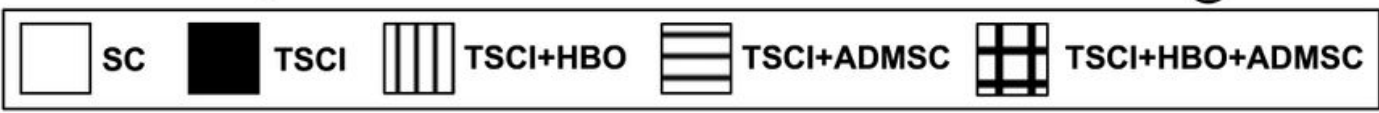

Figure 2

The protein levels of oxidative stress and upstream and downstream inflammatory signalings in spinal cord tissue by day 49 after TSCI procedure A) Protein expression of NOX-1, * vs. other groups with different symbols $(\dagger, \neq, \S), p<0.0001$. B) Protein expression of NOX-2, * vs. other groups with different symbols $(t, \neq, \S), p<0.0001$. C) Protein expression of toll-like receptor (TLR)-4, * vs. other groups with different symbols $(\dagger, \ddagger, \S, 9), p<0.0001$. D) Protein expression of myelin and lymphocyte protein (Mal), * vs. other groups with different symbols $(t, \ddagger, \S), p<0.0001$. E) Protein expression of Myeloid differentiation primary response 88 (MyD88), * vs. other groups with different symbols $(\dagger, \ddagger, \S), p<0.0001$. F) Protein expression of TNF receptor associated factor 6 (TRAF6), * vs. other groups with different symbols $(t, \ddagger, \S), p<0.0001$. G) Protein expression of IKB kinase (IKK)- $a$, ${ }^{*} v s$. other groups with different symbols $(\dagger, \neq, \S, 9), p<0.0001 . H)$ Protein expression of IKK- $\beta,{ }^{*}$ vs. other groups with different symbols ( $\dagger$, 
$\ddagger, \S, 9), p<0.0001$. I) Protein expression of nuclear factor (NF)- $k B$, * vs. other groups with different symbols $(\dagger, \ddagger, \S), p<0.0001$. J) Protein expression of interleukin (IL)- $1 ß, *$ vs. other groups with different symbols $(\dagger, \ddagger, \S, \mathbf{q}), p<0.0001$. K) Protein expression of tumor necrosis factor (TNF)- $a$, ${ }^{*} v s$. other groups with different symbols $(t, \neq, \S), p<0.0001$. L) Protein expression of substance-p, * vs. other groups with different symbols $(t, \ddagger, \S, \mathbf{q}), p<0.0001$. All statistical analyses were performed by one-way ANOVA, followed by Bonferroni multiple comparison post hoc test ( $n=6$ for each group). Symbols $\left({ }^{*}, t, \neq, \S, 9\right)$ indicate significance (at 0.05 level). SC = sham-operated control; $\mathrm{TSCl}=$ traumatic spinal cord injury; HBO = hyperbaric oxygen; $\mathrm{ADMSCs}$ = adipose-derived mesenchymal stem cells.

(A)
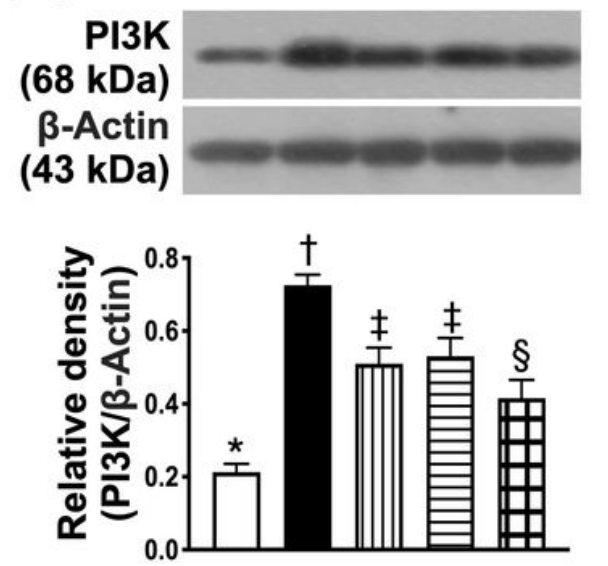

(D)
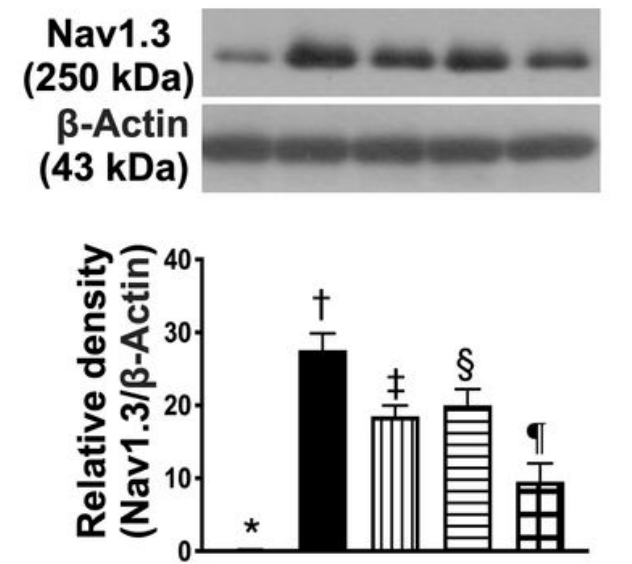

(B)
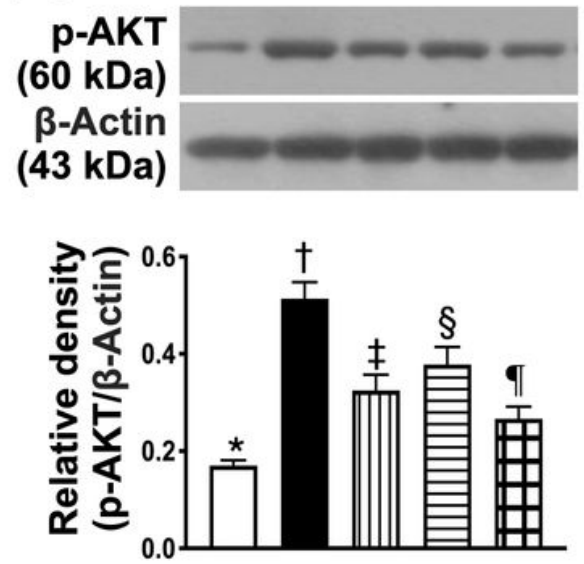

(E)

\section{Nav1.8 \\ (217 kDa) \\ $\beta$-Actin \\ (43 kDa)}

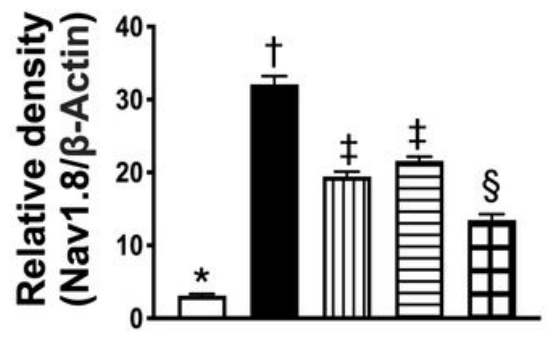

(C)

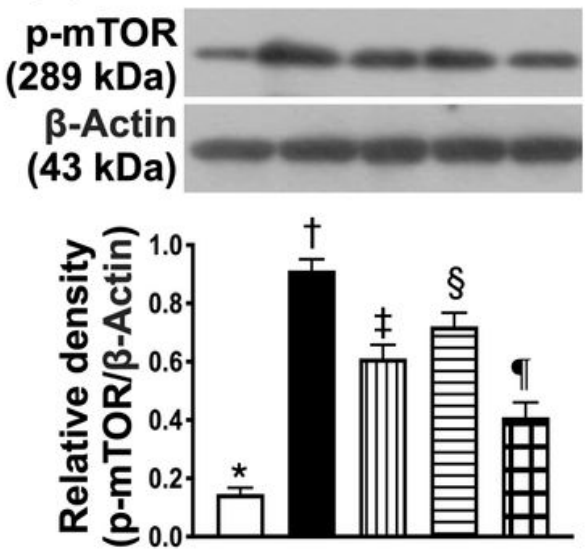

(F)

\section{Nav1.9 \\ (250 kDa) \\ $\beta$-Actin \\ (43 kDa)}



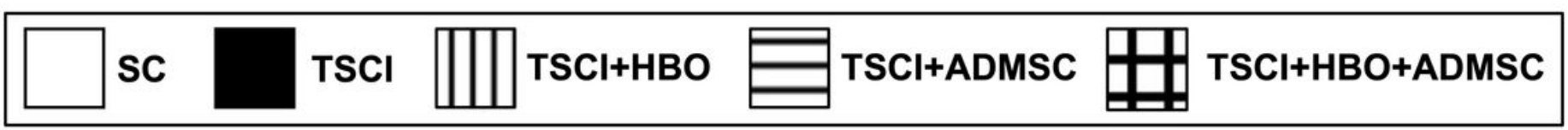

Figure 3

Protein expressions of cell-stress signaling and voltage gated sodium channel in spinal cord tissue by day 49 after TSCI procedure A) Protein expression of PI3K, * vs. other groups with different symbols ( $\dagger$, $\neq$, $\S), p<0.0001$. B) Protein expression of phosphorylated ( $p)$-AKT, * vs. other groups with different symbols $(t, \neq, \S, 9), p<0.0001$. C) Protein expression of $p-m T O R$, * vs. other groups with different symbols $(\dagger, \ddagger, \S$, q), $p<0.0001$. D) Protein expression of Nav1.3, * vs. other groups with different symbols $(\dagger, \ddagger, \S)$, 
$p<0.0001$. E) Protein expression of Nav1.8, * vs. other groups with different symbols $(\dagger, \ddagger, \S), p<0.0001$. F) Protein expression of Nav1.9, * vs. other groups with different symbols $(\dagger, \ddagger, \S), p<0.0001$. All statistical analyses were performed by one-way ANOVA, followed by Bonferroni multiple comparison post hoc test ( $n=6$ for each group). Symbols $(*,+, \neq, \S, 9)$ indicate significance (at 0.05 level). SC = sham-operated control; $\mathrm{TSCl}=$ traumatic spinal cord injury; $\mathrm{HBO}=$ hyperbaric oxygen; $\mathrm{ADMSCs}=$ adipose-derived mesenchymal stem cells.
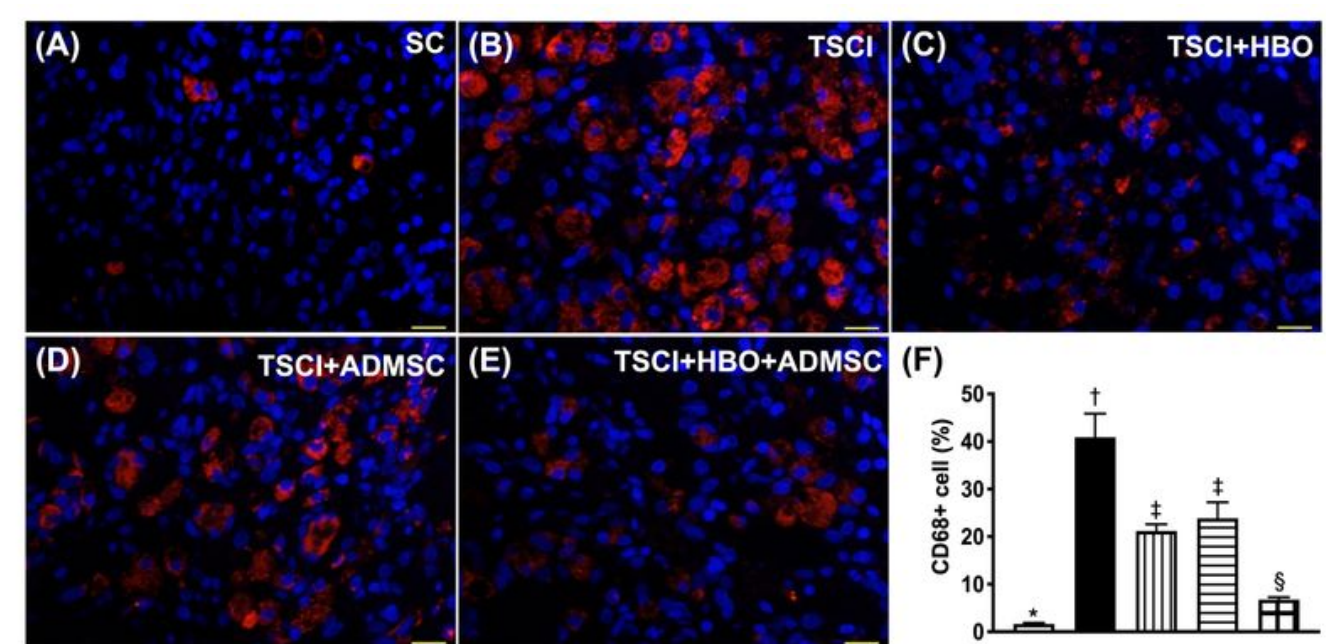

(F)
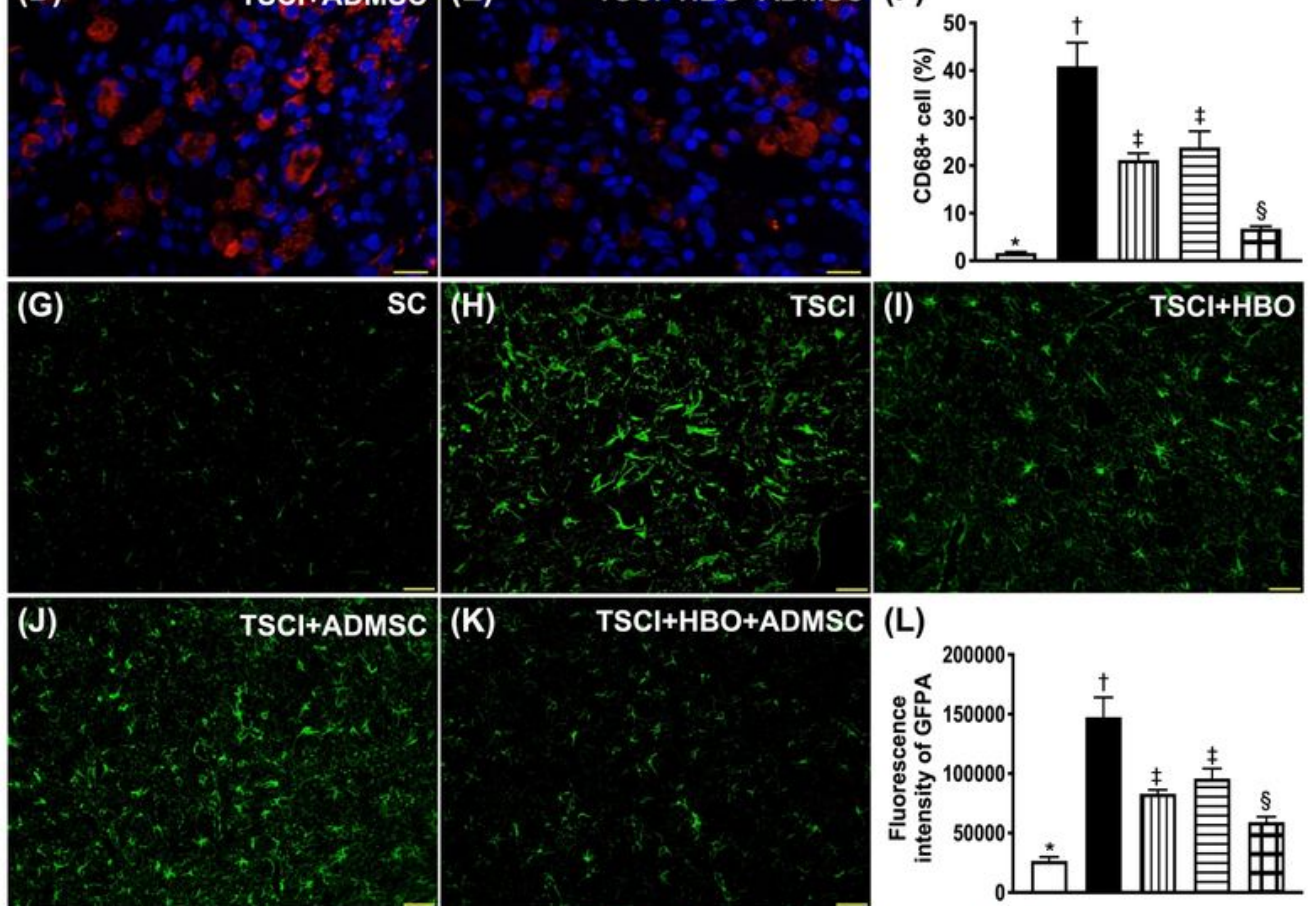

(K)

\section{(L)}
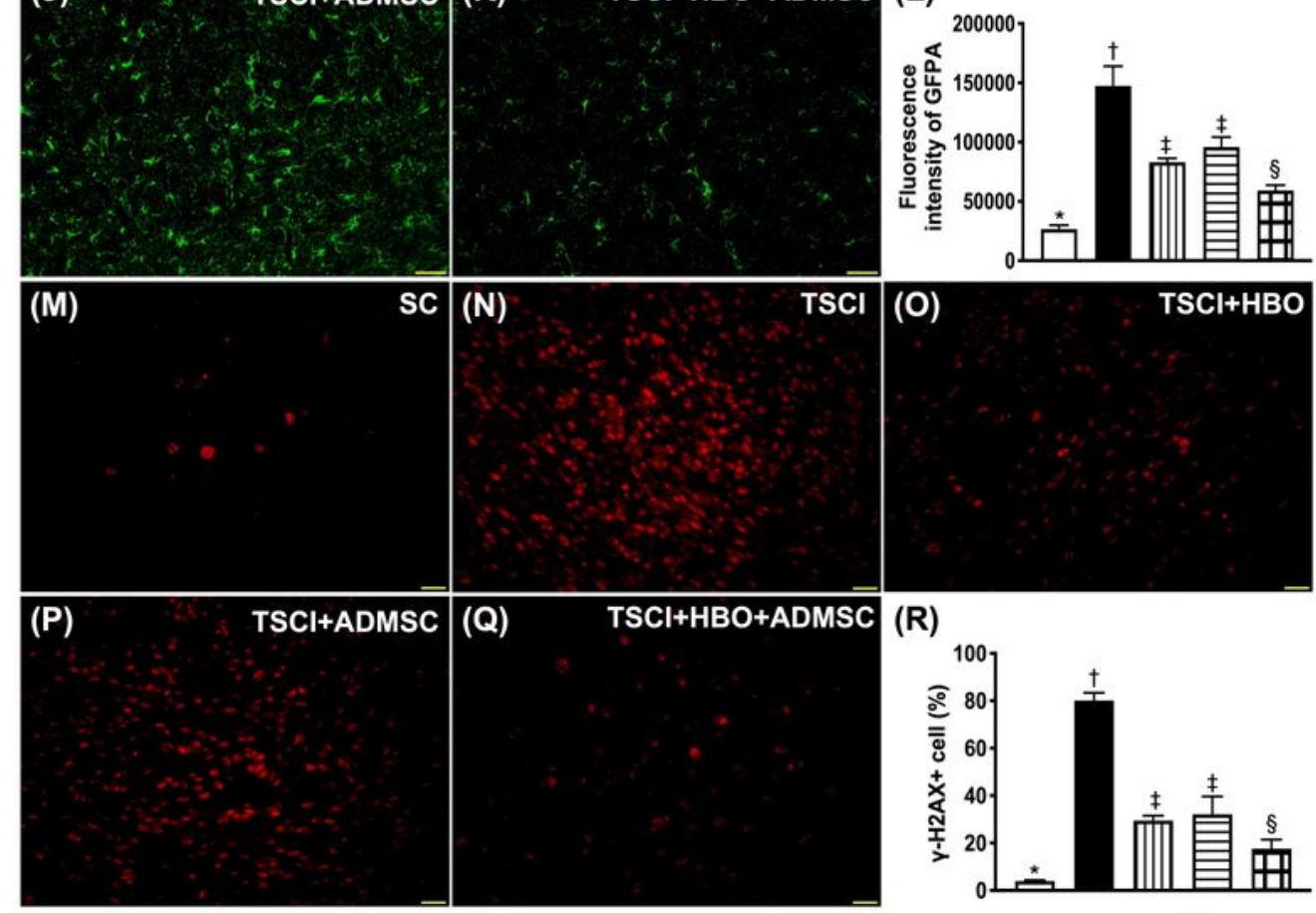

(0)

$\mathrm{TSCl}+\mathrm{HBO}$



(R)

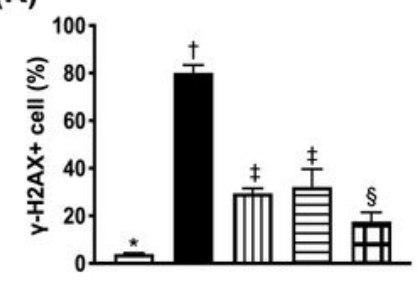

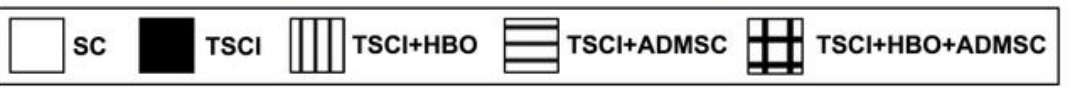

Figure 4 
Cellular levels of inflammation in spinal cord tissue by day 49 after TSCI procedure A to E) Illustrating the immunofluorescent (IF) microscopic finding (400x) for identification of CD68+ cell infiltration in spinal cord (red color). F) Analytical result of percentage of CD68+ cells, * vs. other groups with different symbols $(\dagger, \ddagger, \S), p<0.0001$. G to K) Illustrating the IF microscopic finding (400x) for identification of glial fibrillary acidic protein (GFAP) + cell in spinal cord (green color). L) Analytical result of fluorescent intensity of GFAP+ cells, * vs. other groups with different symbols $(\dagger, \ddagger, \S), p<0.0001$. M to Q) Illustrating the IF microscopic finding (400x) for identification of cellular expression of $\mathrm{y}-\mathrm{H} 2 \mathrm{AX}$ (red color). R) Analytical result of percentage of $\mathrm{y}-\mathrm{H} 2 \mathrm{AX}+$ cells, * vs. other groups with different symbols $(\dagger, \ddagger, \S)$, $p<0.0001$. All scale bars in lower right corner represent $20 \mu \mathrm{m}$. All statistical analyses were performed by one-way ANOVA, followed by Bonferroni multiple comparison post hoc test ( $\mathrm{n}=6$ for each group). Symbols $(*, \dagger, \neq, \S)$ indicate significance (at 0.05 level). SC = sham-operated control; $\mathrm{TSCI}=$ traumatic spinal cord injury; HBO = hyperbaric oxygen; ADMSCs = adipose-derived mesenchymal stem cells.

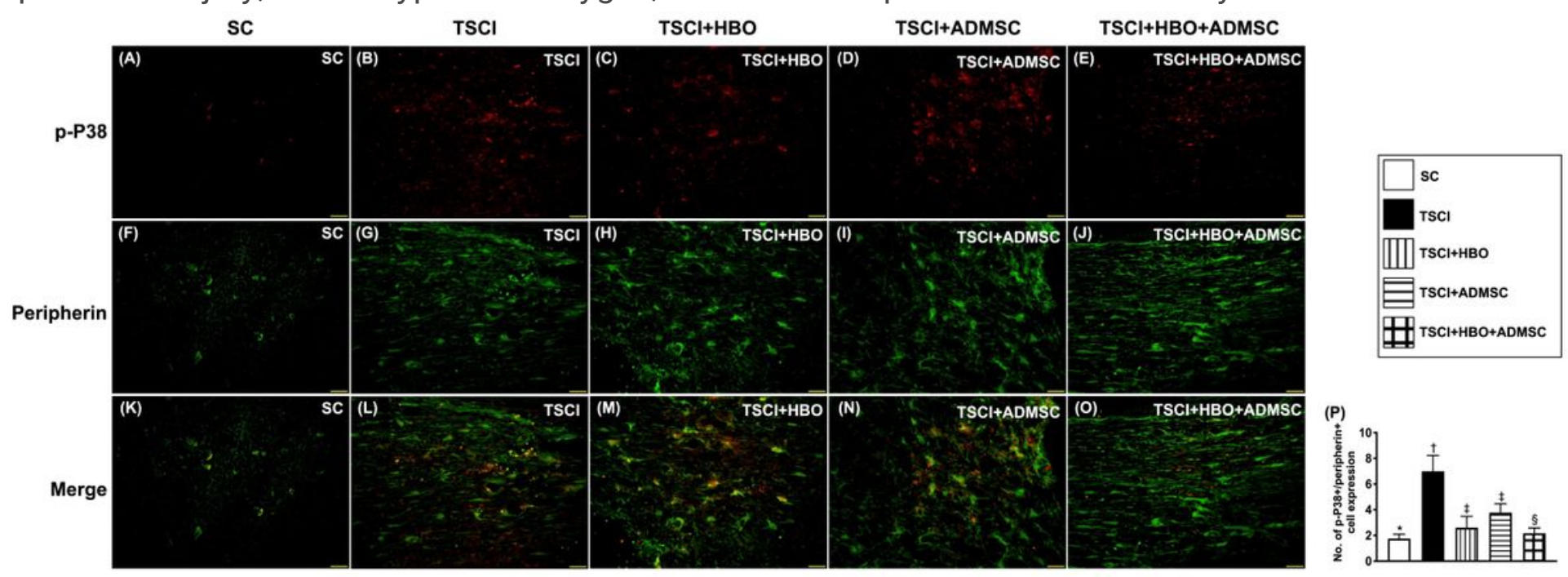

\section{Figure 5}

Immunofluorescent (IF) microscopic finding of colocalization of p-P38 and peripherin in spinal cord neurons by day 49 after TSCI procedure A to E) Illustrating the IF microscopic finding (200x) of positivelystained p-P38 in spinal cord neurons (red color spots). F to J) Illustrating the IF microscopic finding (200x) of positively-stained peripherin cells (green color). $\mathrm{K}$ to $\mathrm{O}$ ) Illustrating the IF microscopic finding (200x) of merged positively-stained p-P38 and peripherin (green-red colocalization). P) Analytical results of number of p-P38+/peripherin+ cells, * denotes statistical significance vs. other groups with different symbols $(\dagger, \ddagger, \S), p<0.0001$. All scale bars in lower right corner represent $20 \mu \mathrm{m}$. All statistical analyses were performed by one-way ANOVA, followed by Bonferroni multiple comparison post hoc test ( $\mathrm{n}=6$ for each group). Symbols $(*,+, \neq, \S)$ indicate significance (at 0.05 level). $S C=$ sham-operated control; $\mathrm{TSCl}=$ traumatic spinal cord injury; $\mathrm{HBO}=$ hyperbaric oxygen; ADMSCs = adipose-derived mesenchymal stem cells. 


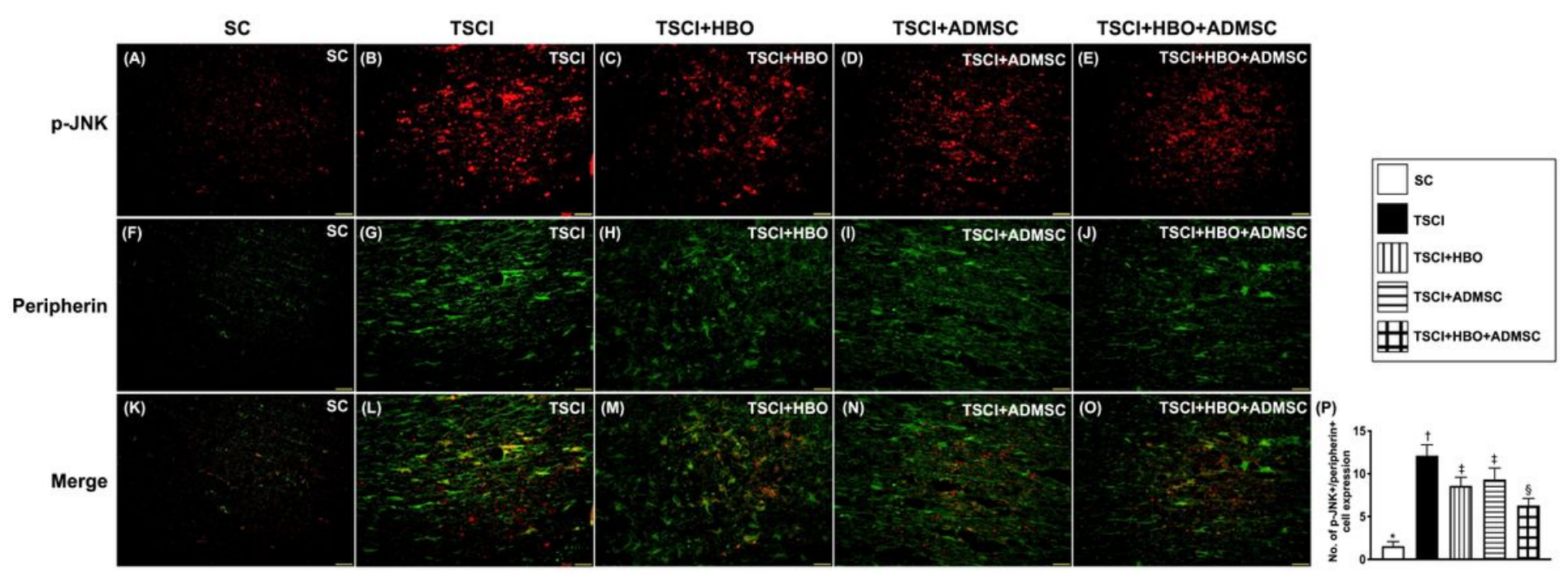

\section{Figure 6}

Immunofluorescent (IF) microscopic finding of colocalization of p-JNK and peripherin in spinal cord neurons by day 49 after TSCI procedure A to E) Illustrating the IF microscopic finding (200x) of positivelystained p-JNK in spinal cord neurons (red color spots). F to J) Illustrating the IF microscopic finding (200x) of positively-stained peripherin cells (green color). $\mathrm{K}$ to 0 ) Illustrating the IF microscopic finding (200x) of merged positively-stained p-JNK and peripherin (green-red colocalization). P) Analytical results of number of p-JNK+/peripherin+ cells, * denotes statistical significance vs. other groups with different symbols $(\dagger, \ddagger, \S), p<0.0001$. All scale bars in lower right corner represent $20 \mu \mathrm{m}$. All statistical analyses were performed by one-way ANOVA, followed by Bonferroni multiple comparison post hoc test $(\mathrm{n}=6$ for each group). Symbols $(*, \uparrow, \ddagger \S)$ indicate significance (at 0.05 level). SC = sham-operated control; $\mathrm{TSCl}=$ traumatic spinal cord injury; $\mathrm{HBO}$ = hyperbaric oxygen; $\mathrm{ADMSCs}$ = adipose-derived mesenchymal stem cells.

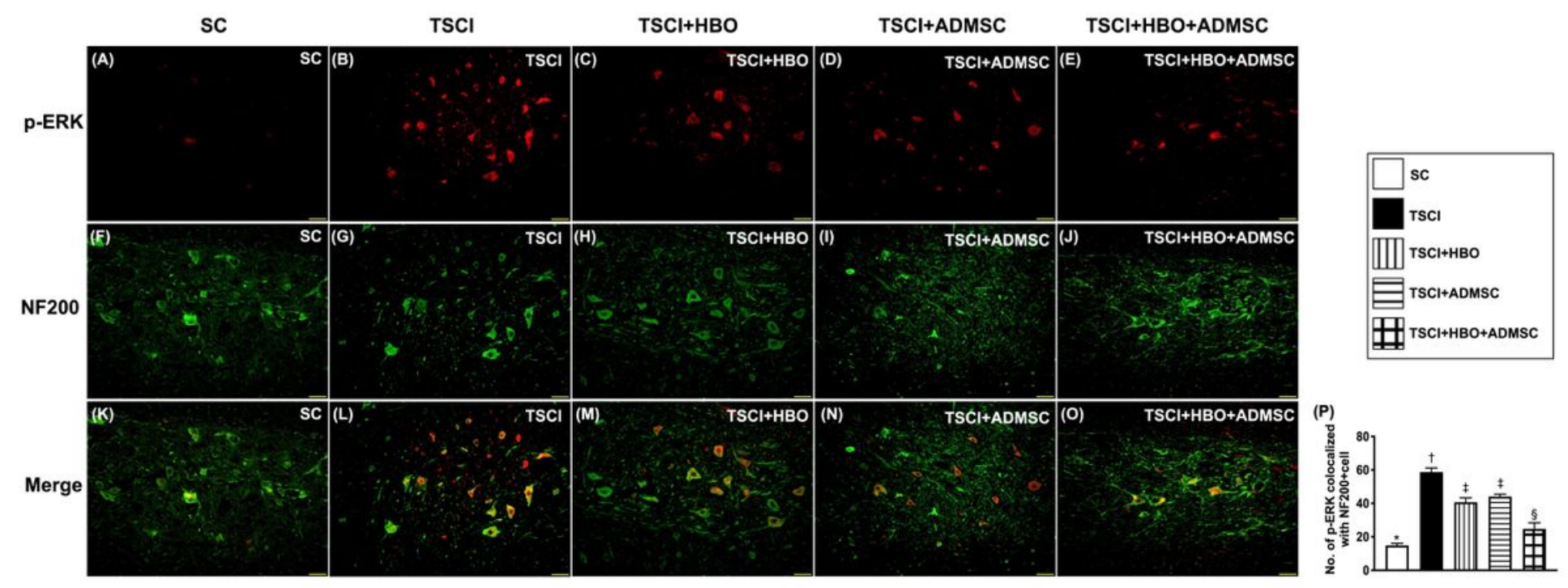

Figure 7 
Immunofluorescent (IF) microscopic finding of colocalization of p-ERK and NF200 in spinal cord neurons by day 49 after TSCI procedure A to E) Illustrating the IF microscopic finding (200x) positively stained pERK in spinal neurons (red color spots). F to J) Illustrating the IF microscopic finding (200x) positively stained NF200 cells (green color). $\mathrm{K}$ to 0 ) Illustrating the IF microscopic finding (200x) of merged positively-stained p-ERK and NF200 (green-red colocalization). P) Analytical results of number of $p$ $\mathrm{ERK}+/ \mathrm{NF200}+$ cells, * denotes statistical significance vs. other groups with different symbols $(\dagger, \ddagger, \S)$, $p<0.0001$. All scale bars in lower right corner represent $20 \mu \mathrm{m}$. All statistical analyses were performed by one-way ANOVA, followed by Bonferroni multiple comparison post hoc test ( $n=6$ for each group). Symbols $(*,+, \neq, \S)$ indicate significance (at 0.05 level). SC = sham-operated control; $\mathrm{TSCl}=$ traumatic spinal cord injury; HBO = hyperbaric oxygen; ADMSCs = adipose-derived mesenchymal stem cells.

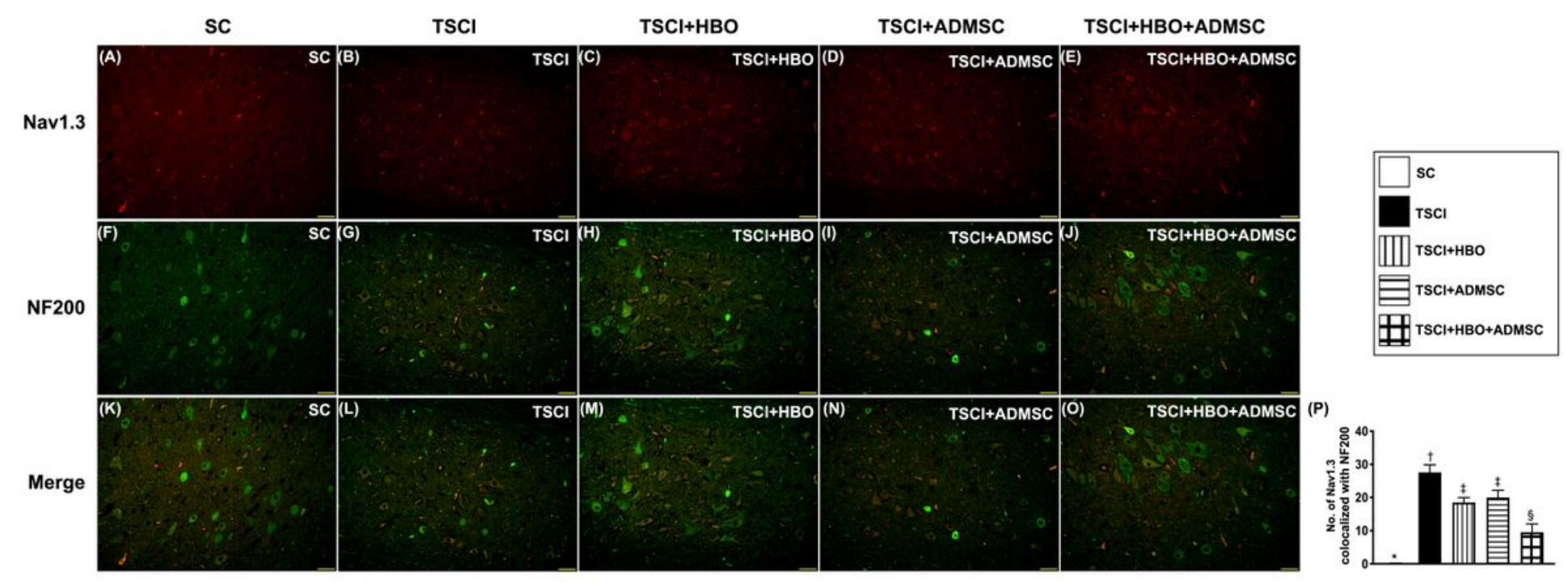

Figure 8

Immunofluorescent (IF) microscopic finding of colocalization of Nav1.3 and NF200 in spinal cord neurons by day 49 after TSCI procedure A to E) Illustrating the IF microscopic finding (200x) positively stained Nav1.3 in spinal neurons (red color spots). F to J) Illustrating the IF microscopic finding (200x) positively stained NF200 cells (green color). K to 0) Illustrating the IF microscopic finding (200x) of merged positively-stained Nav1.3 and NF200 (green-red colocalization). P) Analytical results of number of Nav1.3+/NF200+ cells, * denotes statistical significance vs. other groups with different symbols ( $\uparrow, \neq$,


by one-way ANOVA, followed by Bonferroni multiple comparison post hoc test ( $\mathrm{n}=6$ for each group). Symbols $\left({ }^{*}, \dagger, \ddagger, \S\right)$ indicate significance (at 0.05 level). SC = sham-operated control; $\mathrm{TSCl}=$ traumatic spinal cord injury; HBO = hyperbaric oxygen; ADMSCs = adipose-derived mesenchymal stem cells. 


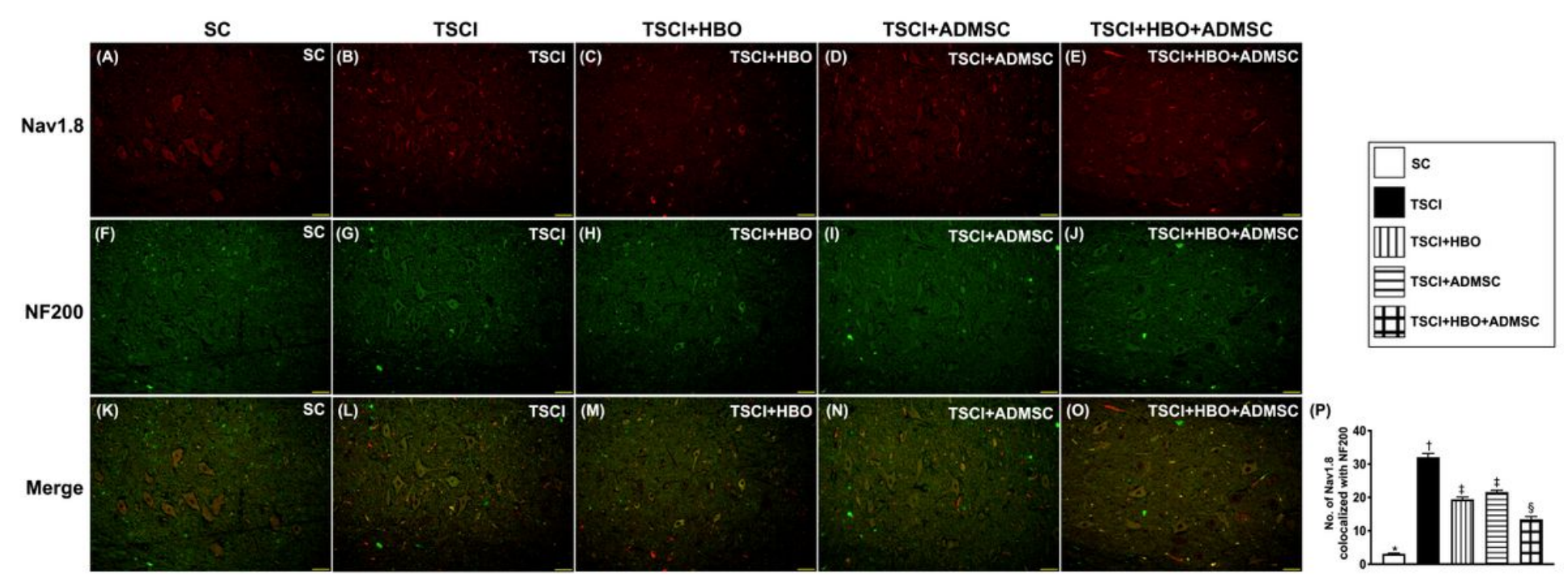

\section{Figure 9}

Immunofluorescent (IF) microscopic finding of colocalization of Nav1.8 and NF200 in spinal cord neurons by day 49 after TSCI procedure A to E) Illustrating the IF microscopic finding (200x) of positively stained Nav1.8 in spinal neurons (red color spots). F to J) Illustrating the IF microscopic finding (200x) of positively stained NF200 cells (green color). K to 0) Illustrating the IF microscopic finding (200x) of merged positively-stained Nav1.8 and NF200 (green-red colocalization). P) Analytical results of number of Nav1.8+/NF200+ cells, * denotes statistical significance vs. other groups with different symbols $(\dagger, \neq$, $\S), p<0.0001$. All scale bars in lower right corner represent $20 \mu \mathrm{m}$. All statistical analyses were performed by one-way ANOVA, followed by Bonferroni multiple comparison post hoc test ( $n=6$ for each group). Symbols $(*,+, \neq, \S)$ indicate significance (at 0.05 level). SC = sham-operated control; $\mathrm{TSCl}=$ traumatic spinal cord injury; $\mathrm{HBO}$ = hyperbaric oxygen; $\mathrm{ADMSCs}$ = adipose-derived mesenchymal stem cells.

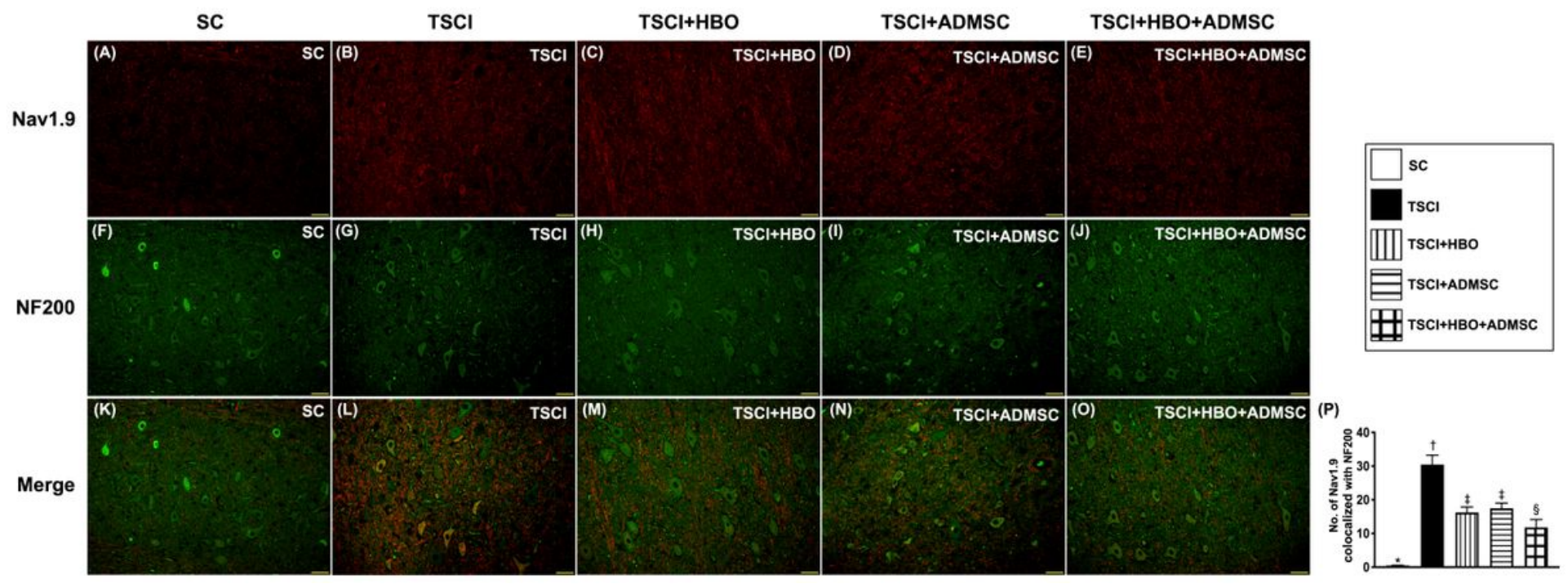

Figure 10 
Immunofluorescent (IF) microscopic finding of colocalization of Nav1.9 and NF200 in spinal cord neurons by day 49 after TSCl procedure A to E) Illustrating the IF microscopic finding (200x) of positively stained Nav1.9 in spinal neurons (red color spots). F to J) Illustrating the IF microscopic finding (200x) of positively stained NF200 cells (green color). K to 0) Illustrating the IF microscopic finding (200x) of merged positively-stained Nav1.9 and NF200 (green-red colocalization). P) Analytical results of number of Nav1.9+/NF200+ cells, * denotes statistical significance vs. other groups with different symbols $(\dagger, \neq$, $\S), p<0.0001$. All scale bars in lower right corner represent $20 \mu \mathrm{m}$. All statistical analyses were performed by one-way ANOVA, followed by Bonferroni multiple comparison post hoc test ( $n=6$ for each group). Symbols $(*, \dagger, \neq, \S)$ indicate significance (at 0.05 level). SC = sham-operated control; $\mathrm{TSCl}=$ traumatic spinal cord injury; $\mathrm{HBO}$ = hyperbaric oxygen; $\mathrm{ADMSCs}$ = adipose-derived mesenchymal stem cells.

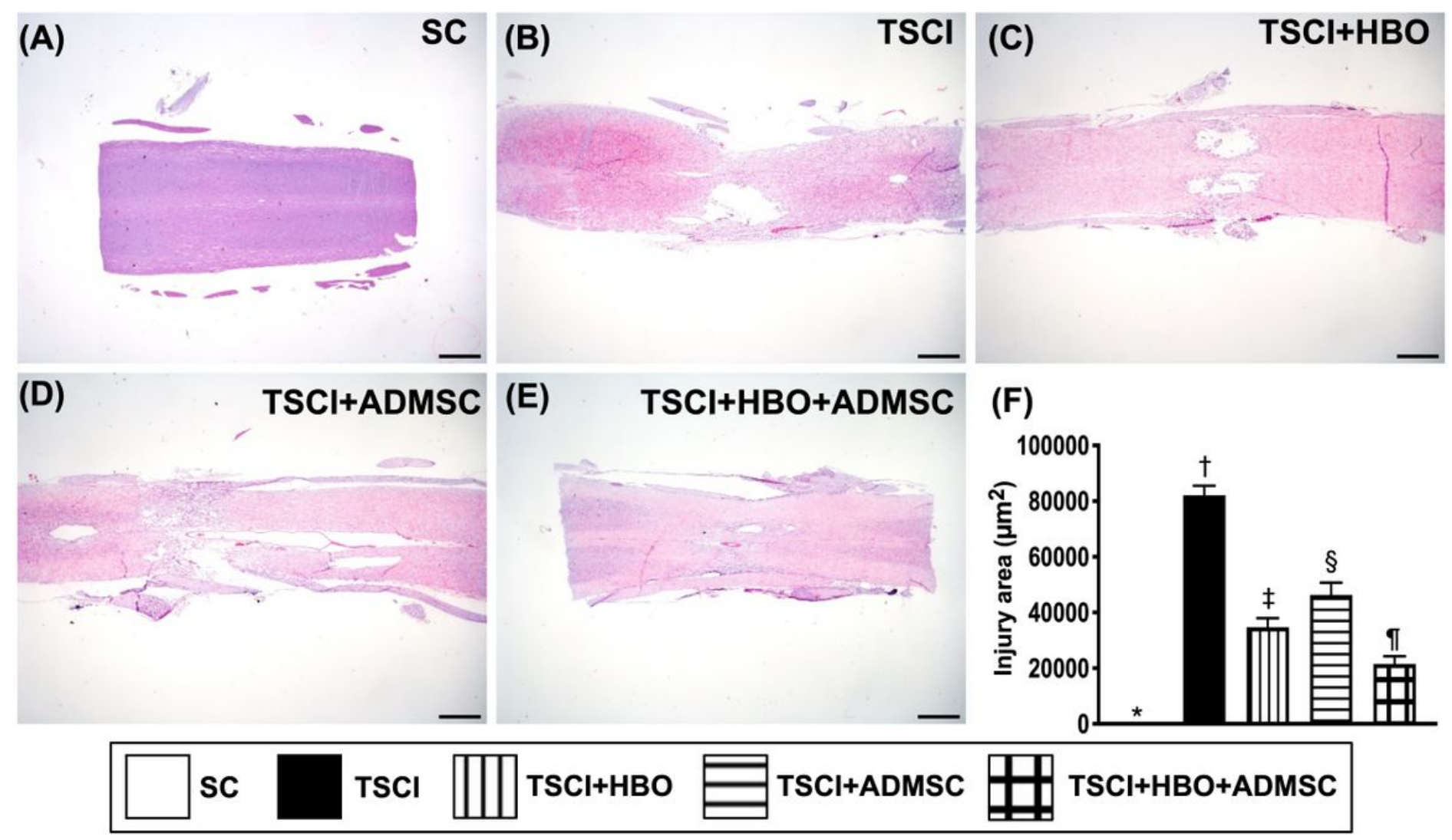

\section{Figure 11}

The spinal cord damaged area by day 49 after TSCI procedure A to E) Illustrating the microscopic finding (10x) of H.E. for identification of spinal cord injured area (red arrows) in longitudinal section. F) Analytical result of spinal cord damaged area, * denotes statistical significance vs. other groups with different symbols $(t, \ddagger, \S), p<0.0001$. All scale bars in lower right corner represent $1.0 \mathrm{~mm}$. All statistical analyses were performed by one-way ANOVA, followed by Bonferroni multiple comparison post hoc test ( $\mathrm{n}=6$ for each group). Symbols $(*, \dagger, \neq, \S)$ indicate significance (at 0.05 level). SC = sham-operated control; $\mathrm{TSCl}=$ traumatic spinal cord injury; $\mathrm{HBO}=$ hyperbaric oxygen; $\mathrm{ADMSCs}$ = adipose-derived mesenchymal stem cells. 


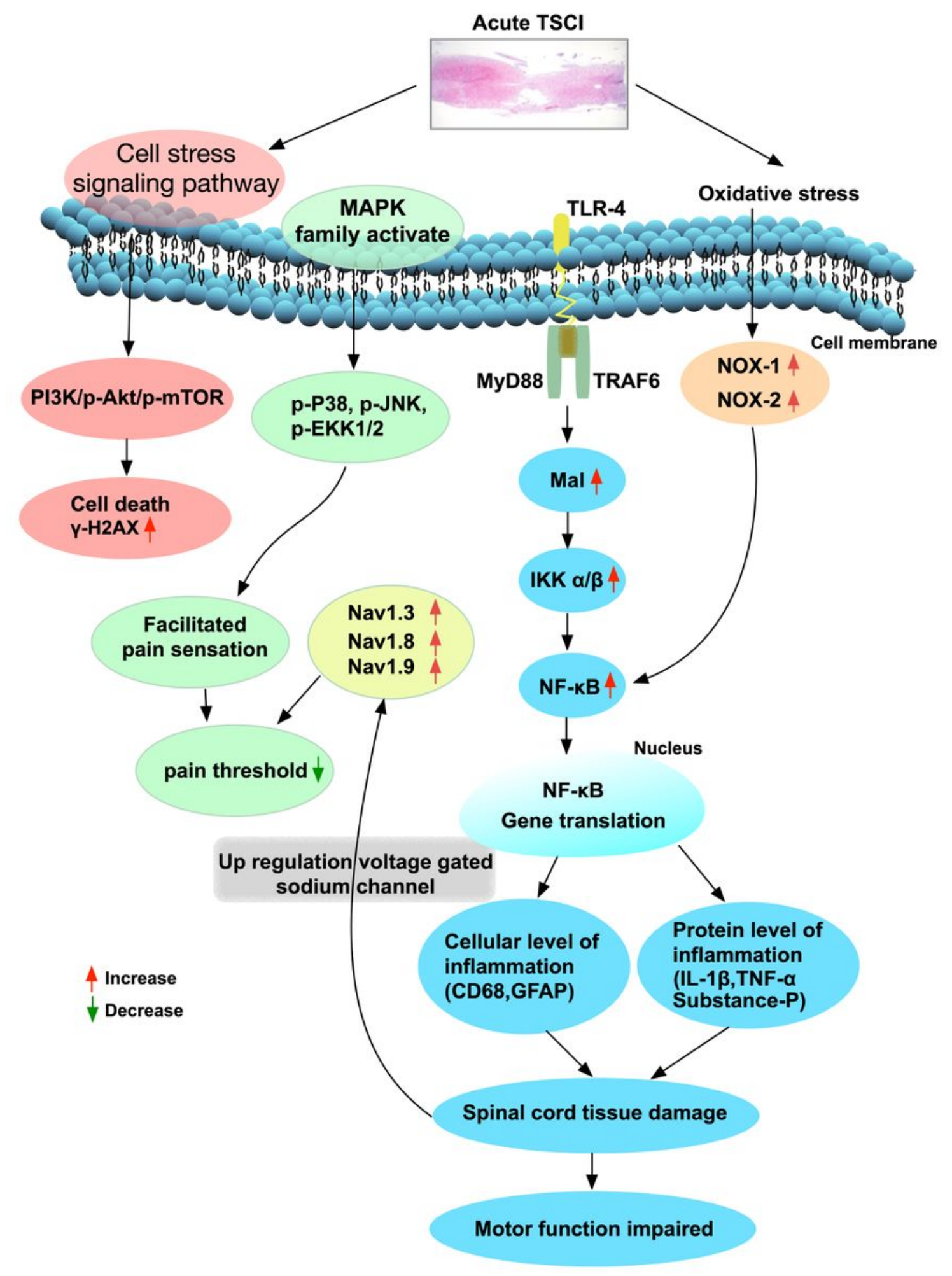

Figure 12

Schematically illustrated the underlying mechanism of signaling pathways activated after acute traumatic spinal cord injury and the effective treatment of HBO-ADMSCs on protecting the neurological function via regulating these signaling pathway $\mathrm{TSCl}$ = traumatic spinal cord injury; three signaling pathways: (1) cell stress signaling; (2) pain facilitated signaling; (3) oxidative stress signaling. 